This is the post peer-review accepted manuscript of:

M. Bin, D. Astolfi, L. Marconi and L. Praly, "About Robustness of Internal ModelBased Control for Linear and Nonlinear Systems," 2018 IEEE Conference on Decision and Control (CDC), Miami Beach, FL, 2018, pp. 5397-5402.

The published version is available online at:

https://doi.org/10.1109/CDC.2018.8619176

(C) 2018 IEEE. Personal use of this material is permitted. Permission from IEEE must be obtained for all other uses, in any current or future media, including reprinting/republishing this material for advertising or promotional purposes, creating new collective works, for resale or redistribution to servers or lists, or reuse of any copyrighted component of this work in other works. 


\title{
About Robustness of Internal Model-Based Control for Linear and Nonlinear Systems
}

\author{
Michelangelo Bin ${ }^{1}$, Daniele Astolfi ${ }^{2}$, Lorenzo Marconi ${ }^{1}$ and Laurent Praly ${ }^{3}$
}

\begin{abstract}
In this paper we propose a general framework in which the robustness properties and requirements of output regulation schemes can be formally described. We introduce a topological definition of robustness relative to arbitrary steady state properties, extending the usual notion of robustness relative to the existence of a steady state in which the regulation error vanishes. We review some of the main control approaches for linear and nonlinear systems, by re-framing their robustness properties within the proposed setting. We show that the celebrated robustness property of the linear regulator, namely the "internal model principle" stated by Francis, Wonham and Davison in the 70's, can be generalized to nonlinear systems in a robustness property relative to the Fourier expansion of the regulation error. We then focus on nonlinear regulation, where we show that only practical regulation can be achieved robustly, while asymptotic regulation is achieved in a quite fragile way. The paper concludes with a conjecture stating that, in a general nonlinear context, asymptotic regulation cannot be achieved in a robust way with a finite dimensional regulator.
\end{abstract}

\section{INTRODUCTION}

The problem of regulating desired outputs to some desired references while rejecting unmeasured disturbances is generically known as output regulation. An elegant solution for linear systems has been given by Francis, Wonham and Davison in the mid 70's, in the seminal works [1], [2]. In that papers, the authors formalized the internal model principle, stating that the property of the error being zero at the steady state is a property that is insensitive to plant parameter variations "only if the controller utilizes feedback of the regulated variable, and incorporates in the feedback path a suitably reduplicated model of the dynamic structure of the exogenous signals which the regulator is required to process". The internal model principle, that is a necessary condition, has been then supported in [2] by a constructive regulator design that actually achieve such celebrated robustness relatively to uncertainties in the plant's parameters. More precisely, the linear regulator of [2] guarantees asymptotic regulation at front of any perturbation of the plant that do not destroy linearity and closed-loop stability. This very powerful and celebrated property has, however, no nonlinear equivalent, in the sense that no existing design

\footnotetext{
${ }^{1}$ Michelangelo Bin and Lorenzo Marconi are with CASY-DEI, University of Bologna (michelangelo.bin2@unibo.it and lorenzo.marconi@unibo.it). Research partially supported by the European Project AirBorne (G.A. 780960).

${ }^{2}$ Daniele Astolfi is with Université de Lorraine, CNRS, CRAN, F54000 Nancy, France (daniele.astolfi@univ-lorraine.fr). Research partially supported by the "Région Grand-Est" of France.

${ }^{3}$ Laurent Praly is with the MINES ParisTech, PSL Research University, CAS - Centre automatique et systémes, Paris 75006, France (Laurent.Praly@mines-paristech.fr).
}

ever provided an extension of that property for arbitrary nonlinear plants and exosystems. Equivalent formulations of the regulation problem and of the internal model principle in a nonlinear case has been proposed at first in a local framework during the late 80's and early 90's (see for instance [3], [4] and the references therein) and successively in a purely nonlinear "non-equilibrium" context (see [5][7]). Nonlinear notions of robustness of the asymptotic regulation property have appeared in the simple case of pure constant disturbances and references (see [8]-[11]), in a local framework where local exponential stability is possible, and in the framework of "structural robustness" [12], where the considered uncertainties are supposed to be concentrated in a finite number of parameters and they are dealt with by immersion arguments (see [12]-[14]). All the other designs dealing with more general systems and exosystems (see e.g. [4], [6], [7], [15]-[17]) provided no robustness results about the property of asymptotic regulation [18], [19] and, up to the authors' knowledge, no existing regulator is able to guarantee asymptotic regulation under arbitrary (even though small and "smooth") nonlinear perturbations of the plant's dynamics. Furthermore, in a nonlinear setting, increasing interest is put in "practical" and "approximate" output regulation problems, where the ideal requirement of having a vanishing regulation error is substituted by an appropriate ultimate bound on it (see for instance [20] and the references therein). The interest of these somewhat "weaker" objectives is motivated by the fact that they require a simpler regulator and they can be achieved in a robust way even for quite general classes of nonlinear systems.

The motivation behind this paper lies in the fact that, while the concept of robustness defined by Francis and Wonham for linear systems has a clear and well-defined meaning, the notion of robustness in a nonlinear setting is still vague, and quite often "robustness results" are claimed in ad-hoc contexts using custom definitions. For instance, the "structural robustness" of [12] extends the "parametric" interpretation of the notion of robustness given by [1], while the perhaps more interesting notion of [10] refers to the "functional" nature of the same definition. Although these two notions are equivalent for linear functions between finitedimensional vector spaces, they do not lead to equivalent notions if nonlinear functions are considered. As a matter of fact, if $f: \mathbb{R}^{n} \rightarrow \mathbb{R}^{n}$ is linear, then fixing a basis of $\mathbb{R}^{n}$ defines a function $m$ that sends $f$ to its matrix representation $F=m(f) \in \mathbb{R}^{n \times n}$. We may consider parametric variations of $f$ by "moving" $F=m(f)$ in $\mathbb{R}^{n \times n}$ (thus a total of $n^{2}$ parameters). If $|\cdot|$ is a norm on $\mathbb{R}^{n \times n}$, these variations are 
quantified by looking at the norm $\left|F-F^{\circ}\right|$ of the deviation of $F$ relatively to its nominal value $F^{\circ}$, so as variations in the same neighbourhood of $F^{\circ}$ are quantified equally. If we induce a topology on the space $\mathcal{F}$ of linear maps $\mathbb{R}^{n} \rightarrow \mathbb{R}^{n}$ by completing the collection of subsets of the form $m^{-1}(V)$, where $V$ is a neighbourhood in the topology induced by $|\cdot|$ on $\mathbb{R}^{n \times n}$, then we obtain a topology on $\mathcal{F}$ which is equivalent to the one considered in [10] (the $C^{1}$ topology [21]), and that is in direct relation with the parametric interpretation of the matrix variations. If $f$ is not linear, however, this correspondence is not true anymore. For instance, consider the family $\mathcal{F}$ of functions of the kind $f_{\mu}(x)=\mu x^{2}$, with $\mu$ a parameter ranging in an interval $I \subset \mathbb{R}$. While for any $\mu_{1}, \mu_{2} \in I, f_{\mu_{1}}$ and $f_{\mu_{2}}$ belong to the same class $\mathcal{F}$, the function $g(x)=f_{\mu}(x)+x^{3}$ does not belong to $\mathcal{F}$ for any choice of $\mu \in I$. While $f_{\mu_{1}}$ and $f_{\mu_{2}}$ are obtained in the spirit of the "structural" notion of [12], $g$ is obtained in the "differential topology" spirit of [10], and they lead to totally different concept of variation.

In this paper we aim at proposing a unifying concept of robustness relying of a general topological notion of "variation" that includes all the previous cases as particular examples. We also aim at extending the notion of robustness to properties more general than "asymptotic regulation', by capturing in this way a wider variety of "robust behaviours" exhibited by practical and approximate regulation designs. We show that the robustness property of the linear regulator can be framed in the language of this paper and it naturally extends to a milder robustness condition when applied to nonlinear systems. We also review the general design of [7] for nonlinear systems and we show that robustness of asymptotic regulation does not hold for smooth plant's variations, while a practical regulation property does. We end the paper with a conjecture that says that no (finite-dimensional) regulator can ensure the same robustness property of the linear regulator in a nonlinear context.

Notation and Preliminary Notions: $\mathbb{R}$ denotes the set of real numbers, $\mathbb{N}$ denotes the set of non-negative integers, and $\emptyset$ denotes the empty set. If $x$ belongs to a normed space, we denote by $|x|$ its norm. A topology $\tau$ on a set $S$ is a family of subsets of $S$ satisfying: $i$ ) $\emptyset, S \in \tau$; ii) $\tau$ is closed under finite and infinite unions; iii) $\tau$ is closed under finite intersections. The elements of $\tau$ are called open sets. If $s \in S$, a subset $U \subset S$ is called a $\tau$-neighbourhood of $s$ if it contains an open set containing $s$. With $S$ a subset of a topological space, we denote by $\mathcal{K}(S)$ the set of all the compact subsets of $S$. $\operatorname{int}(S)$ denotes the interior of a set $S$.

Consider a system of the form

$$
\mathcal{H}: \dot{x}=f(x)
$$

defined over a normed vector space $\mathbb{X}$, with $f$ a continuously differentiable function. Given any subset $X \subset \mathbb{X}$, we call $\mathcal{S}_{\mathcal{H}}(X)$ the set of maximal solutions to (1) originating in $X$. For $T \geq 0$ we define the $T$-reachable set of (1) from $X$ as

$$
\mathcal{R}_{\mathcal{H}}^{T}(X):=\left\{x(t) \in \mathbb{X}: x \in \mathcal{S}_{\mathcal{H}}(X), t \geq T\right\}
$$

and the $\Omega$-limit set of $X$ as

$$
\Omega_{\mathcal{H}}(X):=\lim _{T \rightarrow \infty} \mathcal{R}_{\mathcal{H}}^{T}(X)=\bigcap_{T \geq 0} \mathcal{R}_{\mathcal{H}}^{T}(X) .
$$

We say that $\mathcal{H}$ is uniformly eventually bounded from $X$ if exists $T \geq 0$ such that $\mathcal{R}_{\mathcal{H}}^{T}(X)$ is bounded. The following result summarizes the main properties of $\Omega_{\mathcal{H}}(X)$.

Proposition $1 \Omega_{\mathcal{H}}(X)$ exists and is closed. If $\mathcal{H}$ is uniformly eventually bounded from $X, \Omega_{\mathcal{H}}(X)$ is compact, non empty, invariant, uniformly attractive from $X$ and is the smallest (in the sense of inclusion) closed set with this latter property.

Proposition 1 is a direct consequence of the definitions (2)(3) and of the group property the flow of (1). Proposition 1, and in particular the fact that $\Omega_{\mathcal{H}}(X)$ is the smallest set with such properties, motivates referring to $\Omega_{\mathcal{H}}(X)$ as the steady state locus of the trajectories of (1) originating in $X$.

\section{Framework And Problem Statement}

Consider a controlled system described by a nominal model of the form

$$
\dot{x}=f^{o}(w, x, u) \quad y=h^{o}(w, x)
$$

where $x \in \mathbb{R}^{n}$ is the state, $u \in \mathbb{R}^{m}$ is the control input $y \in \mathbb{R}^{p_{y}}$, is the measured output, and the functions $\left(f^{o}, h^{o}\right)$ are supposed to be smooth enough. The measured output $y$ is partitioned as $y:=\operatorname{col}\left(e, y_{m}\right)$ in which $e \in \mathbb{R}^{p_{e}}$, with $p_{e} \leq p_{y}$, denotes a regulation error on which there are "asymptotic performance expectations", as detailed later, and $y_{m} \in \mathbb{R}^{p-p_{e}}$ are possible extra measurements. The input $w \in \mathbb{R}^{n_{w}}$ is supposed to be a bounded external signal that may represent a reference to be tracked or a disturbance to be rejected. As customarily done in output regulation theory, in this work we suppose that $w$ is generated by an autonomous system, denoted as exosystem, of the form

$$
\dot{w}=s(w)
$$

although, most of the considerations reported below don't necessarily rely upon this assumption.

System (4) represents a nominal model on which we argue that an output feedback controller of the form

$$
\dot{\eta}=\phi(\eta, y), \quad u=\theta(\eta, y), \quad \eta \in \mathbb{R}^{n_{\eta}}
$$

has been designed so that the resulting closed-loop system satisfies certain properties. For instance, in the problem of asymptotic output regulation it is required that the trajectories of the resulting closed-loop system (4)-(6), with initial conditions $(x(0), \eta(0))$ and exogenous signal $w(t)$ ranging in some prescribed sets, are bounded and the associated regulation error $e(t)$ is asymptotically vanishing. Depending on the sets where the initial conditions and the exogenous signal are assumed to range, global, semiglobal, or local output regulation problems can be indeed formulated (see, for instance, [3] and references therein). Other problem formulations that are closely related with this paper can be stated in terms of non-exact output regulation, in which the error generated by the closed-loop dynamics is required to be 
asymptotically bounded by not necessarily zero values. This relaxed condition can be of particular interest in order to deal with complex nonlinear systems that make the asymptotic requirement hard to be achieved, unless introducing limiting "immersion" assumptions (see e.g. [6], [15]). In this respect, a further distinction may be done between practical output regulation problems, in which the asymptotic bound of the error is fixed a priory as an arbitrary number, and approximate output regulation problems, in which no bound for the asymptotic error is a priory fixed and the objective is to achieve the "best" one can expect from the actual problem with the adopted design tools. See [12], [13], [16], [19], [22][25], for some examples. In this paper we generically denote by $\mathbf{P}$ a property expected on the asymptotic regulation error and we say that the "regulation objective $\mathbf{P}$ " is achieved by a regulator (6) if the error trajectories associated to the closedloop system asymptotically satisfy the property $\mathbf{P}$. Examples of properties are clearly "error identically zero" as in the asymptotic output regulation problem or "error with bounded amplitude" in the non-exact version, or other properties that will be specified throughout the paper.

The problem of designing a regulator so that a regulation objective $\mathbf{P}$ is fulfilled assumes a conceptual and practical relevance as soon as robustness aspects are taken into account, namely as soon as the regulation objective must be guaranteed not only in nominal conditions, namely when the regulated system behaves as nominal dynamics (4), but also when parametric or structural uncertainties are present. In the following, we thus consider the case in which the real (unknown) model of the controlled system has the form

$$
\dot{x}=f(w, x, u), \quad y=h(w, x)
$$

in which $(f(\cdot, \cdot, \cdot), h(\cdot, \cdot))$ are obtained by "perturbing" the nominal model (4). We consider the problem in which the regulation objective $\mathbf{P}$ is required to hold, not only for the nominal system (4), but also for all possible actual dynamics (7) that are "close enough" to (4), in some sense that will be made precise below. Namely, the property $\mathbf{P}$ is preserved under plant perturbations.

\section{A Robustness Definition}

For convenience we will consider exogenous signals $w$ that are solutions of an exosystem of the kind (5). The extended plant, given by (5) and (7), is thus defined by the functions $F:=(s, f, h)$ and the regulator (6) is designed to enforce a given property $\mathbf{P}$ on the steady-state trajectories under the assumption that $F$ equals a nominal value $F^{\circ}:=\left(s^{\circ}, f^{\circ}, h^{\circ}\right)$ and the initial conditions range in a nominal set $W_{0}^{\circ} \times X_{0}^{\circ} \subset$ $\mathbb{R}^{n_{w}} \times \mathbb{R}^{n}$. This yields a definition of the maps $\phi$ and $\theta$ in (6) and of a set $H_{0} \subset \mathbb{R}^{n_{\eta}}$ of initial conditions for $\eta$. In order to study the "robustness" features of the regulator relatively to the property $\mathbf{P}$ and under variation of the plant's function $F$ and of the initial set $W_{0} \times X_{0}$, in the following we consider a closed-loop system of the form

$$
\begin{aligned}
\dot{w} & =s(w) \\
\mathcal{H}_{F}: & \dot{x}=f(w, x, \theta(\eta, h(w, x))) \\
\dot{\eta} & =\phi(\eta, h(w, x))
\end{aligned}
$$

in the case in which the actual plant's function $F$ and the actual initial set $W_{0} \times X_{0}$ possibly differ from the nominal values. For simplicity, in the following we let $\mathbf{x}:=(w, x)$ and $\mathbf{n}=\operatorname{dim}(\mathbf{x})$. We restrict the attention to functions $F$ that belong to a given functional space $\mathcal{F}$, that we equip with a topology $\tau_{\mathcal{F}}$, and to compact sets of initial conditions that belong to $\mathcal{X}:=\mathcal{K}\left(\mathbb{R}^{\mathbf{n}}\right)$, that we endow with the Hausdorff topology ${ }^{1} \tau_{\mathcal{X}}$. The particular value of $\mathcal{F}$ and $\tau_{\mathcal{F}}$ will be specified later depending on the context. With reference to the notation introduced at the end of Section I, we denote by $\mathcal{S}_{F}\left(\mathbf{X}_{0} \times H_{0}\right)$ and $\Omega_{F}\left(\mathbf{X}_{0} \times H_{0}\right)$ the quantities $\mathcal{S}_{\mathcal{H}_{F}}\left(\mathbf{X}_{0} \times H_{0}\right)$ and $\Omega_{\mathcal{H}_{F}}\left(\mathbf{X}_{0} \times H_{0}\right)$ obtained with a given $F \in \mathcal{F}$ and a given $\mathbf{X}_{0} \subset \mathbb{R}^{\mathbf{n}}$. We equip the product space $\mathcal{F} \times \mathcal{X}$ with the product topology $\tau_{\mathcal{F} \times \mathcal{X}}$ and we make the notion of "perturbation" $\left(F, \mathbf{X}_{0}\right)$ of $\left(F^{\circ}, \mathbf{X}_{0}^{\circ}\right)$ precise by the following definition.

Definition $1\left(F, \mathbf{X}_{0}\right) \in \mathcal{F} \times \mathcal{X}$ is a perturbation of $\left(F^{\circ}, \mathbf{X}_{0}^{\circ}\right)$ if it belongs to a $\tau_{\mathcal{F} \times \mathcal{X}}$-neighbourhood of $\left(F^{\circ}, \mathbf{X}_{0}^{\circ}\right)$.

The regulation objective has been previously introduced throughout the informal definition of a property $\mathbf{P}$ that we wish the trajectories of the closed-loop system to asymptotically have. With $\mathbb{S}$ the set of all the functions $\mathbb{R}_{+} \rightarrow \mathbb{R}^{\mathbf{n}} \times \mathbb{R}^{n_{\eta}}$, we can formally define the property $\mathbf{P}$ by associating to its informal statement the subset $\mathcal{P} \subset \mathbb{S}$ given by $\mathcal{P}:=\{(\mathbf{x}, \eta) \in \mathbb{S}: \mathbf{P}$ holds $\}$. We then say that $(\mathbf{x}, \eta) \in \mathbb{S}$ has the property $\mathbf{P}$ if $(\mathbf{x}, \eta) \in \mathcal{P}$. To formally express what we mean by saying that the property P should hold "asymptotically", we restrict our attention to the trajectories of (8) that originates in the attractor $\Omega_{F}\left(\mathbf{X}_{0} \times H_{0}\right)$. In order to work with a well-defined (in the sense of Proposition 1) set $\Omega_{F}\left(\mathbf{X}_{0} \times H_{0}\right)$, we also need as a basic robustness ingredient that the regulator (6) guarantees that $\mathcal{H}_{F}$ has the desired boundedness properties for the considered perturbations. We put all together within the following definition.

Definition 2 With $\left(F, \mathbf{X}_{0}\right) \in \mathcal{F} \times \mathcal{X}$, we say that the regulator (6) achieves the regulation objective $\mathbf{P}$ asymptotically at $\left(F, \mathbf{X}_{0}\right)$ if $\mathcal{H}_{F}$ is uniformly eventually bounded from $\mathbf{X}_{0} \times H_{0}$ and $(\mathbf{x}, \eta) \in \mathcal{S}_{F}\left(\Omega_{F}\left(\mathbf{X}_{0} \times H_{0}\right)\right)$ implies $(\mathbf{x}, \eta) \in \mathcal{P}$.

Let $V \subset \mathcal{F} \times \mathcal{X}$, then we say that $V$ generates an equibounded family of systems ${ }^{2}$ if for every $\left(F, \mathbf{X}_{0}\right) \in V$ the system $\mathcal{H}_{F}$ defined as in (8) is uniformly eventually bounded from $\mathbf{X}_{0} \times H_{0}$ and there exists a compact set $\mathcal{O} \subset \mathbb{R}^{\mathbf{n}}$ such that $\Omega_{F}\left(\mathbf{X}_{0} \times H_{0}\right) \subset \mathcal{O}$ for all $\left(F, \mathbf{X}_{0}\right) \in V$. We define now a formal notion of robustness for the regulator (6) associated to the property $\mathbf{P}$.

Definition 3 We say that the regulator (6) is $\mathbf{P}$-robust at $\left(F^{\circ}, \mathbf{X}_{0}^{\circ}\right)$ with respect to $\tau_{\mathcal{F}}$ if there exists a $\tau_{\mathcal{F} \times \mathcal{X}^{-}}$ neighbourhood $V$ of $\left(F^{\circ}, \mathbf{X}_{0}^{\circ}\right)$ that generates an equibounded family of systems such that, for all $\left(F, \mathbf{X}_{0}\right) \in V$, the

\footnotetext{
${ }^{1}$ Namely, the topology induced by the distance function: $d\left(\mathbf{X}_{1}, \mathbf{X}_{2}\right)=\max \left\{\sup _{\mathbf{s u n}_{1} \in \mathbf{X}_{1} \inf _{2} \in \mathbf{X}_{2}}\left|\mathbf{x}_{1}-\mathbf{x}_{2}\right|, \sup _{\mathbf{x}_{2} \in \mathbf{X}_{2}} \inf _{1} \in \mathbf{X}_{1}\left|\mathbf{x}_{2}-\mathbf{x}_{1}\right|\right\}$ ${ }^{2}$ Equiboundedness is needed to avoid unfortunate limit cases in which there exists a sequence $\left(\left(F^{n}, \mathbf{X}_{0}^{n}\right)\right)_{n}$ in $V$ such that the corresponding sequence $\left(\Omega_{F^{n}}\left(\mathbf{X}_{0}^{n} \times H_{0}\right)\right)_{n}$ escapes to the horizon.
} 
regulator achieves the regulation objective $\mathbf{P}$ asymptotically at $\left(F, \mathbf{X}_{0}\right)$.

\section{Robustness in Regulation Schemes With LINEAR INTERNAL MODEL}

In this section we consider the class of regulators (6) obtained by partitioning the state $\eta$ as $\eta=\left(\eta_{\mathrm{im}}, \eta_{\mathrm{s}}\right)$, with $\eta_{\mathrm{im}} \in \mathbb{R}^{n_{\mathrm{im}}}$ and $\eta_{\mathrm{s}} \in \mathbb{R}^{n_{\mathrm{s}}}$ that satisfy the dynamic equations

$$
\begin{aligned}
& \dot{\eta}_{\mathrm{im}}=\Phi \eta_{\mathrm{im}}+G e \\
& \dot{\eta}_{\mathrm{s}}=\phi_{\mathrm{s}}\left(\eta_{\mathrm{s}}, \eta_{\mathrm{im}}, y\right) \\
& u=\theta(\eta, y) .
\end{aligned}
$$

By following [10], in this paper we focus on "smooth variations" of $F$. More precisely, with $\mathbf{X} \subset \mathbb{R}^{\mathbf{n}}$ and $\mathbf{U} \subset$ $\mathbb{R}^{m}$ arbitrarily large compact sets, throughout this section we suppose that $F$ ranges in the set $\mathcal{C}^{1}(\mathbf{X} \times \mathbf{U})$ of all the continuously differentiable functions defined on $\mathbf{X} \times \mathbf{U}$ and with values in $\mathbb{R}^{\mathbf{n}} \times \mathbb{R}^{p}$. We endow $\mathcal{C}^{1}(\mathbf{X} \times \mathbf{U})$ with the weak topology [21] $\tau_{\mathcal{C}^{1}}$ defined as follows: with $\ell:=$ $\max \left\{\max _{\mathbf{x} \in \mathbf{X}}|\mathbf{x}|, \max _{\mathbf{u} \in \mathbf{U}}|\mathbf{u}|\right\}$ and for any $F \in \mathcal{C}^{1}(\mathbf{X} \times \mathbf{U})$ and $\epsilon>0$, an $\epsilon$-neighbourhood of $F$ is given as

$$
\begin{array}{r}
\mathcal{N}_{\epsilon}(F)=\left\{G \in \mathcal{C}^{1}(\mathbf{X} \times \mathbf{U}): \max _{\mathbf{p} \in \mathbf{X} \times \mathbf{U}}|F(\mathbf{p})-G(\mathbf{p})|<\ell \epsilon,\right. \\
\left.\max _{\mathbf{p} \in \mathbf{X} \times \mathbf{U}}\left|F^{\prime}(\mathbf{p})-G^{\prime}(\mathbf{p})\right|<\epsilon\right\},
\end{array}
$$

where $F^{\prime}$ and $G^{\prime}$ denote the derivative of $F$ and $G$. We stress that $\mathbf{X}$ and $\mathbf{U}$ are arbitrary and can be chosen large enough to encompass all the solutions of interest. Restricting the functions $F$ to $\mathbf{X} \times \mathbf{U}$ though allows us to consider a nicer topology $\tau_{\mathcal{C}^{1}}$ which is first-countable and metrizable rather than the alternative strong topology (see [21]).

\section{A. Robustness of the Linear Regulator}

We start considering the case in which $F^{\circ} \in \mathcal{C}^{1}(\mathbf{X} \times \mathbf{U})$ is linear and the linear regulator (see e.g. [1], [2]) is used. The regulator is obtained from (9) by: a) letting $H_{0} \in \mathcal{K}\left(\mathbb{R}^{n_{\eta}}\right)$ be arbitrary, b) letting $\left(\phi_{\mathrm{s}}(\cdot), \theta_{\mathrm{s}}(\cdot)\right)$ be linear functions whose matrix representation with respect to a fixed basis of $\mathbb{R}^{\mathbf{n}} \times$ $\mathbb{R}^{n_{\eta}}$ is of the kind

$$
\begin{aligned}
\dot{\eta}_{\mathrm{s}} & =A_{s} \eta_{\mathrm{s}}+B_{s 1} \eta_{\mathrm{im}}+B_{s 2} y \\
u & =K_{1} \eta+K_{2} y
\end{aligned}
$$

c) choosing $(\Phi, G)$ as any controllable pair with $\Phi$ that has a characteristic polynomial which coincides with the minimal polynomial of the corresponding matrix representation of $s^{\circ}(\cdot)$ and, finally, d) by fixing $A_{s}, B_{s 1}, B_{s 2}, K_{1}, K_{2}$ so as to stabilise the nominal system $\mathcal{H}_{F} \circ$ originating from $\mathbf{X}_{0}^{\circ} \times H_{0}$.

We let $\mathcal{F}_{L} \subset \mathcal{C}^{1}(\mathbf{X} \times \mathbf{U})$ be the set of all the linear functions in $\mathcal{C}^{1}(\mathbf{X} \times \mathbf{U})$ and we let

$$
\mathcal{F}:=\left\{F \in \mathcal{F}_{L}: s=s^{\circ}\right\} .
$$

We endow $\mathcal{F}$ with the subset topology $\tau_{\mathcal{F}}$ derived by $\left(\mathcal{C}^{1}(\mathbf{X} \times \mathbf{U}), \tau_{\mathcal{C}^{1}}\right)$ and we let $\mathbf{P}_{0}$ the property

$$
\mathbf{P}_{0}=" e=h_{e}(w, x)=0 " .
$$

The next result, that follows from [2], captures the main robustness property of the linear regulator.
Proposition 2 The linear regulator (10) is $\mathbf{P}_{0}$-robust at $\left(F^{\circ}, \mathbf{X}_{0}^{\circ}\right)$ with respect to $\tau_{\mathcal{F}}$.

Actually, a stronger result can be given: the $\mathbf{P}_{0}$-robustness property of Proposition 2 is universal in $\mathbf{X}_{0}$, i.e. the same regulator achieves $\mathbf{P}_{0}$ for any initial condition of the plant. Nevertheless, although the linear regulator is $\mathbf{P}_{0}$-robust for "C ${ }^{1}$ variations" of linear functions that let $s^{\circ}$ unchanged, this property is a mere consequence of linearity and it is broken by any slight nonlinear perturbation of $F$ or by any (even linear) perturbation of $s$. More precisely, if instead of (11) we consider a larger sets of the kind $\mathcal{F}=\mathcal{F}_{L}$ or $\mathcal{F}=\left\{F \in \mathcal{C}^{1}(\mathbf{X} \times \mathbf{U}): s=s^{\circ}\right\}$, endowed with the correspondent subset topologies, then Proposition 2 does not apply anymore and, as a simple counter-example can easily show, the property of $\mathbf{P}_{0}$-robustness is lost.

\section{B. Robustness in the $\mathbf{P}_{T}$ Sense}

The limits of the $\mathbf{P}_{0}$-robustness of the linear regulator motivate seeking for a regulation objective $\mathbf{P}$ for which a regulator that is $\mathbf{P}$-robust relatively to more general topological spaces $\left(\mathcal{F}, \tau_{\mathcal{F}}\right)$ more likely could be constructed. As proposed in [23], in this section we let $\mathcal{F}=\mathcal{C}^{1}(\mathbf{X} \times \mathbf{U})$, $\tau_{\mathcal{F}}=\tau_{\mathcal{C}^{1}}$ and we consider a regulator of the kind (9) with $(\phi, \theta)$ possibly nonlinear and with $n_{\mathrm{im}}=(2 d+1) p_{e}$, for some arbitrary $d \in \mathbb{N}$. We then choose a basis for $\mathbb{R}^{\mathbf{n}} \times \mathbb{R}^{n_{\eta}}$ in which $(\Phi, G)$ read as

$$
\Phi=\left(\begin{array}{ccccc}
0 & I & 0 & \ldots & 0 \\
0 & 0 & I & \cdots & 0 \\
\vdots & & & \ddots & \vdots \\
0 & & & & I \\
-a_{1} I & -a_{2} I & \ldots & & -a_{2 d+1} I
\end{array}\right) \quad G=\left(\begin{array}{c}
0 \\
0 \\
\vdots \\
0 \\
I
\end{array}\right)
$$

with $a_{1}, \ldots, a_{2 d+1}$ chosen so that the characteristic polynomial of $\Phi$ is

$$
p_{\Phi}(\lambda)=\lambda \cdot \prod_{k=1}^{d}\left(\lambda^{2}+\omega_{k}^{2}\right),
$$

where we let $\omega_{k}:=2 \pi k / T$ for some $T \geq 0$. For a given continuous function $\alpha: \mathbb{R}_{+} \rightarrow \mathbb{R}$, let

$$
\mathrm{c}_{k}(\alpha):=\int_{0}^{T} \alpha(\nu) e^{-i 2 \pi k \nu / T} d \nu
$$

be the Fourier coefficient corresponding to the $k$-th harmonic $2 \pi k / T$ and let

$$
\mathcal{Q}_{d}:=\left\{\alpha: \mathbb{R} \rightarrow \mathbb{R}: c_{k}(\alpha)=0, k=0, \ldots, d\right\}
$$

be the subspace of the functions $\mathbb{R} \rightarrow \mathbb{R}$ that have null harmonics at $\omega_{k}=2 \pi k / T$. We define the regulation objective:

$$
\mathbf{P}_{T}^{\text {weak }}=" \eta \text { is not T-periodic or } e \in\left(\mathcal{Q}_{d}\right)^{p_{e}} "
$$

and, with $F^{\circ} \in \mathcal{C}^{1}(\mathbf{X} \times \mathbf{U})$ and $\mathbf{X}_{0}^{\circ} \in \mathcal{X}$, we make the following assumption

A1) There exists a $\tau_{\mathcal{C}^{1} \times \mathcal{X}}$-neighbourhood $V$ of $\left(F^{\circ}, \mathbf{X}_{0}^{\circ}\right)$ that generates an equibounded family of systems. 
Then the following result holds.

Proposition 3 Assume A1. Then the regulator is $\mathbf{P}_{T}^{\text {weak }}$ robust at $\left(F^{\circ}, \mathbf{X}_{0}^{\circ}\right)$ with respect to $\tau_{\mathcal{C}^{1}}$.

Proposition 3 states that, as long as a steady-state is defined, either the closed-loop solutions converge to a solution where $\eta$ is not periodic or asymptotically $e$ has null mean value and null harmonics at $\omega_{k}, k=1, \ldots, d$. We can refine the result under additional assumption concluding $\mathbf{P}_{T}$-robustness where

$$
\mathbf{P}_{T}=" e \in\left(\mathcal{Q}_{d}\right)^{p_{e}} " .
$$

Let $z:=\operatorname{col}(x, \eta)$ and let $g: \mathbb{R}^{n_{w}} \times \mathbb{R}^{n} \times \mathbb{R}^{n_{\eta}} \rightarrow \mathbb{R}^{n+n_{\eta}}$ be such that (8) with $F=F^{\circ}$ can be rewritten as

$$
\dot{w}=s(w), \quad \dot{z}=g(w, z) .
$$

Then the following holds.

Proposition 4 Assume that the z subsystem is 0-locally exponentially stable (0-LES) and the origin of the $w$ subsystem is stable. Suppose, moreover, that there exists a $\tau_{\mathcal{C}^{1} \times \mathcal{X}^{-}}$ neighbourhood $V$ of $\left(F^{\circ},\{0\}\right)$ such that for all $\left(F, \mathbf{X}_{0}\right) \in V$ and all $(\mathbf{x}, \eta) \in \mathcal{S}_{F}\left(\mathbf{X}_{0} \times H_{0}\right)$, $w$ is T-periodic. Then the regulator is $\mathbf{P}_{T^{-}}$-robust at $\left(F^{\circ},\{0\}\right)$ with respect to $\tau_{\mathcal{C}^{1}}$.

The proof of Proposition 4 follows from the fact that if $z$ is 0-LES and $w$ is periodic and sufficiently small then the closed loop trajectories are periodic as well, and hence $\mathbf{P}_{T}^{\text {weak }}$ implies $\mathbf{P}_{T}$. Details are omitted for reason of space. Another refinement of Proposition 3 is given as follows.

Proposition 5 Assume that Al holds for some $\tau_{\mathcal{F} \times \mathcal{X}}$ neighbourhood $V$ of $\left(F^{\circ}, \mathbf{X}_{0}^{\circ}\right)$ and suppose that, for each $\left(F, \mathbf{X}_{0}\right) \in V$ there exist a class-L function $\beta$ and a class-K functions $\rho$ such that each two solutions $\left(w_{1}, x_{1}, \eta_{1}\right),\left(w_{2}, x_{2}, \eta_{2}\right) \in \mathcal{S}_{F}\left(\mathbf{X}_{0} \times H_{0}\right)$ fulfil

$\left|\left(x_{1}(t), \eta_{1}(t)\right)-\left(x_{2}(t), \eta_{2}(t)\right)\right| \leq \beta(t)+\rho\left(\left|w_{1}(t)-w_{2}(t)\right|\right)$

for all $t \in \mathbb{R}_{+}$. Suppose in addition that, for each solution $\left(w^{\star}, x^{\star}, \eta^{\star}\right)$ in $\mathcal{S}_{F}\left(\Omega_{F}\left(\mathbf{X}_{0} \times H_{0}\right)\right)$, w is T-periodic. Then the regulator is $\mathbf{P}_{T}$-robust at $\left(F^{\circ}, \mathbf{X}_{0}^{\circ}\right)$ with respect to $\tau_{\mathcal{F}}$.

\section{Quasi-Periodic Robustness}

We can deal with quasi-periodic responses in the same way as in the previous section. In the following we use the same regulator as in Section IV-B, with $(\Phi, G)$ given by (13) and with (14) that still holds, with the $\omega_{k}$ 's that are arbitrarily chosen in $\mathbb{R}_{+}$and where we also let for convenience $\omega_{0}:=0$. With $\alpha: \mathbb{R} \rightarrow \mathbb{R}_{+}$and for $k=0, \ldots, d$, let us define the (generalised) Fourier coefficients as

$$
c_{k}^{\prime}(\alpha):=\lim _{T \rightarrow \infty} \frac{1}{T} \int_{0}^{T} \alpha(\nu) e^{-i \omega_{k} \nu} d \nu
$$

and let

$$
\mathcal{Q}_{d}^{\prime}:=\left\{\alpha: \mathbb{R} \rightarrow \mathbb{R}: c_{k}^{\prime}(\alpha)=0, k=0, \ldots, d\right\} .
$$

Proceeding as in Section IV-B, we let

$$
\mathbf{P}_{q p}^{\text {weak }} ;=" \eta \text { is not quasi-periodic or } e \in\left(\mathcal{Q}_{d}^{\prime}\right)^{p_{e}} " .
$$

Then the following result holds.

Proposition 6 Assume Al. Then the regulator is $\mathbf{P}_{q p}^{\text {weak }}$ robust at $\left(F^{\circ}, \mathbf{X}_{0}^{\circ}\right)$ with respect to $\tau_{\mathcal{C}^{1}}$.

\section{Robustness in Nonlinear Regulation Schemes}

In this section we focus on output regulation for nonlinear systems. For simplicity, we refer to the framework of [7], however the same conclusions apply to [15] and to the related extensions of the frameworks (see for instance [13], [26] [28]). We thus limit to the SISO case, in which $y=e$ and $m=p_{y}=p_{e}=1$, and where the state of the plant can be decomposed as $x=\operatorname{col}(z, e)$, with $z \in \mathbb{R}^{n-1}$, and with $f(\cdot)$ that is defined so as the plant has the following structure

$$
\begin{aligned}
& \dot{z}=g(w, z, e) \\
& \dot{e}=q(w, z, e)+b(w, z, e) u
\end{aligned}
$$

for some $g: \mathbb{R}^{n_{w}} \times \mathbb{R}^{n} \rightarrow \mathbb{R}^{n-1}, q, b: \mathbb{R}^{n_{w}} \times \mathbb{R}^{n} \rightarrow \mathbb{R}$ and with $h(w, x)=e$. The regulator design is made assuming that the initial conditions of (16) range in an arbitrary compact set $W \times Z \times E \subset \mathbb{R}^{n_{w}} \times \mathbb{R}^{n}$, which we will assume fixed from now on. In line with the previous sections, we let $\mathbf{X} \in \mathcal{K}\left(\mathbb{R}^{n_{w}} \times \mathbb{R}^{n}\right)$ and $\mathbf{U} \in \mathcal{K}(\mathbb{R})$ be arbitrarily large compact sets such that $W \times Z \times E \subset \mathbf{X}$ and with $\mathbf{U}$ taken sufficiently large to encompass all the solutions of interest. We also adapt the definitions of $\mathcal{C}^{1}(\mathbf{X} \times \mathbf{U})$ and $\tau_{\mathcal{C}^{1}}$ to this case accordingly. As basic assumptions on the plant's data, we assume the following

A2) $W$ is invariant for the exosystem $w$.

A3) There exist functions $\pi: W \rightarrow \mathbb{R}^{n}, u^{\star}: W \rightarrow \mathbb{R}^{m}$ solving the regulator equations

$$
\begin{aligned}
\frac{\partial \pi(w)}{\partial w} s(w) & =g(w, \pi(w), 0), \\
0 & =q(w, \pi(w), 0)+b(w, \pi(w), 0) u^{\star}(w) .
\end{aligned}
$$

A4) The set graph $\pi$ is asymptotically stable ${ }^{3}$ for the system

$$
\dot{w}=s(w), \quad \dot{z}=g(w, z, 0)
$$

with a domain of attraction including $W \times Z$.

As the structure of (16), the invariance of $W$, the existence of $\left(\pi, u^{\star}\right)$ and the minimum phase assumption are properties of $F=(s, f, h)$, we let $\mathcal{F}$ be the set of functions in $\mathcal{C}^{1}(\mathbf{X} \times$ $\mathrm{U})$ for which these properties holds.

The functions $\phi(\cdot)$ and $\theta(\cdot)$ of (6) are chosen so as

$$
\begin{aligned}
& \dot{\eta}=F \eta+G(\gamma(\eta)+\kappa(e)) \\
& u=\gamma(\eta)+\kappa(e),
\end{aligned}
$$

with $H_{0}$ arbitrary, $(F, G)$ a controllable pair and with $n_{\eta} \in \mathbb{N}, \gamma: \mathbb{R}^{n_{\eta}} \rightarrow \mathbb{R}$ and $\kappa: \mathbb{R} \rightarrow \mathbb{R}$ that are continuous functions chosen on the basis of $W, Z, E$ and $F^{\circ} \in \mathcal{F}$ by following the procedure of [7]. The set $H_{0} \subset \mathbb{R}^{n_{\eta}}$ is instead chosen arbitrarily. The main result of [7] can be framed in the language of this paper as follows.

Proposition 7 Let $\tau_{\mathcal{F}}$ be any topology containing $\left\{F^{\circ}\right\}$. With $\mathbf{P}_{0}$ defined as in (12), $F^{\circ} \in \mathcal{F}$ and $\mathbf{X}_{0}^{\circ}:=W_{0}^{\circ} \times$ $Z_{0}^{\circ} \times E_{0}^{\circ} \subset \operatorname{int}(W \times Z \times E)$, the regulator (17) is $\mathbf{P}_{0}$-robust at $\left(F^{\circ}, \mathbf{X}_{0}^{\circ}\right)$ with respect to $\tau_{\mathcal{F}}$.

\footnotetext{
${ }^{3}$ This is indeed a minimum phase assumption. For further details the reader is referred to [7].
} 
In other words, Proposition 7, which is indeed the result of [7], states that the $\mathbf{P}_{0}$-robustness is proved only for perturbations of $\left(F^{\circ}, \mathbf{X}_{0}^{\circ}\right)$ that keep $F^{\circ}$ constant and $\mathbf{X}_{0}^{\circ}$ inside the set $W \times Z \times E$. As a matter of fact, as $\mathbf{X}_{0}^{\circ}$ is in the interior of $W \times Z \times E$, we can always find a $\tau_{\mathcal{X}^{-}}$ neighbourhood of $\mathbf{X}_{0}^{\circ}$ that stays inside $W \times Z \times E$. Hence to construct the $\tau_{\mathcal{F} \times \mathcal{X}}$-neighbourhood $V$ of Definition 3 we just need to find a $\tau_{\mathcal{F}}$-neighbourhood (call it $N$ ) of $F^{\circ}$ for which $\mathbf{P}_{0}$ holds. The result of [7] states indeed that the choice $N=\left\{F^{\circ}\right\}$ works. The fact that also slight $\mathcal{C}^{1}$ perturbations of $F^{\circ}$ might destroy the property $\mathbf{P}_{0}$ can be verified by means of simple counter-examples. Even though a general result on the fragility of $\mathbf{P}_{0}$ with respect to general $\mathcal{C}^{1}$ variations is not known at present, we believe that it holds true. In particular, we are led to believe that the property $\mathbf{P}_{0}$ is in its nature "nominal" and cannot be preserved, in general, under arbitrary (even if small) variations. This belief is included as a particular case in the forthcoming conjecture stated in Section VI.

Robustness to more general variations is instead possible when an "approximate" regulation goal is considered. More precisely, for any $\varepsilon>0$, let $\mathbf{P}_{\varepsilon}="|e|_{\infty}<\varepsilon$ ". Let $\tau_{\mathcal{F}}$ be now the subset topology induced by $\left(\mathcal{C}^{1}(\mathbf{X} \times \mathbf{U}), \tau_{\mathcal{C}^{1}}\right)$, and let the regulator (6) be chosen so that $P_{\varepsilon}$ holds at $\left(F^{\circ}, \mathbf{X}_{0}^{\circ}\right)$, with $F^{\circ} \in \mathcal{F}$ and $\mathbf{X}_{0}^{\circ} \subset \operatorname{int}(W \times Z \times E)$ compact. Then the following result comes as a consequence of the continuity of $F, \gamma$ and $\kappa$.

Proposition 8 The regulator (16) is $\mathbf{P}_{\varepsilon}$-robust at $\left(F^{\circ}, \mathbf{X}_{0}^{\circ}\right)$ with respect to $\tau_{\mathcal{F}}$.

\section{CONCLUSIONS}

In this paper we dealt with the robustness issue in output regulation schemes. We proposed a general definition of robustness relative to a steady-state property that generalises the classical regulation goal of reaching a steady state in which the regulation errors are identically zero. We reviewed some of the main regulation schemes in linear and nonlinear frameworks, by showing, in relevant cases, what kind of robustness properties are meet. Starting from the special $\mathbf{P}_{0}$-robustness property of the linear regulator, throughout the milder $\mathbf{P}_{T}$-robustness for nonlinear systems, up to the weak robustness property of nonlinear design of Proposition 7 , we arrived to the claim that $\mathbf{P}_{0}$-robustness might not be the most appropriate goal in general nonlinear regulation. We conclude this paper with the conjecture below, stating that, even if the exosystem is known, no finite-dimensional regulator can guarantee asymptotic regulation under general " $\mathrm{C}^{1}$ variations" of the plant. With $s^{\circ}$ the nominal (known) exosystem function and with $\mathbf{X} \in \mathcal{K}\left(\mathbb{R}^{n_{w}} \times \mathbb{R}^{n}\right)$ and $\mathbf{U} \in \mathcal{K}\left(\mathbb{R}^{m}\right)$ arbitrary compact sets, let $\mathcal{F}:=\left\{F \in \mathcal{C}^{1}(\mathbf{X} \times \mathbf{U}): s=s^{\circ}\right\}$ and let $\tau_{\mathcal{F}}$ be the subset topology derived by $\left(\mathcal{C}^{1}(\mathbf{X} \times \mathbf{U}), \tau_{\mathcal{C}^{1}}\right)$. Then we state the following.

Conjecture 1 Let $F^{\circ} \in \mathcal{F}$ and $\mathbf{X}_{0}^{\circ} \in \mathcal{K}\left(\mathbb{R}^{n_{w}} \times \mathbb{R}^{n}\right)$. Then no regulator of the kind (6) is $\mathbf{P}_{0}$-robust at $\left(F^{\circ}, \mathbf{X}_{0}^{\circ}\right)$ with respect to $\tau_{\mathcal{F}}$.

\section{REFERENCES}

[1] B. A. Francis and W. M. Wonham. The internal model principle of control theory. Automatica, 12:457-465, 1976.

[2] E. J. Davison. The robust control of a servomechanism problem for linear time-invariant multivariable systems. IEEE Trans. on Automat. Cont., 21:25-34, 1976.

[3] C. I. Byrnes and A. Isidori. Output regulation for nonlinear systems: an overview. Int. J. Robust Nonlinear Control, 10:323-337, 2000.

[4] J. Huang. Nonlinear output regulation: theory and applications. Siam, 2004.

[5] C. I. Byrnes, A. Isidori, and A. Praly. On the asymptotic properties of a system arising from the non-equilibrium theory of output regulation. Mittag Leffler Institute, 2003.

[6] C. I. Byrnes and A. Isidori. Limit sets, zero dynamics and internal models in the problem of nonlinear output regulation. IEEE Trans. on Automat. Cont., 48:1712-1723, 2003.

[7] L. Marconi, L. Praly, and A. Isidori. Output stabilization via nonlinear Luenberger observers. SIAM Journal of Control and Optimization, 45:2277-2298, 2007.

[8] H. K. Khalil. Universal integral controllers for minimum-phase nonlinear systems. IEEE Trans. on Automat. Cont., 45:490-494, 2000.

[9] F. Poulain and L. Praly. Robust asymptotic stabilization of nonlinear systems by state feedback. In Proc. 8th IFAC NOLCOS, pages 653658, 2010.

[10] D. Astolfi and L. Praly. Integral action in output feedback for multiinput multi-output nonlinear systems. IEEE Trans. on Automat. Cont., 62(4):1559-1574, 2017.

[11] X. Huang, H. K. Khalil, and Y. Song. Regulation of non-minimumphase nonlinear systems using slow integrators and high-gain feedback. IEEE Transactions on Automatic Control, 2018. to appear.

[12] C. Byrnes, F. Delli Priscoli, and A. Isidori. Structurally stable output regulation for nonlinear systems. Automatica, 33(3):369-385, 1997.

[13] A. Isidori, L. Marconi, and L. Praly. Robust design of nonlinear internal models without adaptation. Automatica, 48:2409-2419, 2012.

[14] M. Bin, D. Astolfi, and L. Marconi. Robust internal model design by nonlinear regression via low-power high-gain observers. In 2016 IEEE 56th Conference on Decision and Control (CDC), Las Vegas, USA, 2016.

[15] C. I. Byrnes and A. Isidori. Nonlinear internal models for output regulation. IEEE Trans. on Automat. Cont., 49:2244-2247, 2004.

[16] L. Marconi and L. Praly. Uniform practical nonlinear output regulation. IEEE Trans. on Automat. Cont., 53:1184-1202, June 2008.

[17] M. Lu and J. Huang. A class of nonlinear internal models for global robust output regulation problem. International Journal of Robust Nonlinear Control, 25:1831-1843, 2015.

[18] M. Bin and L. Marconi. The chicken-egg dilemma and the robustness issue in nonlinear output regulation with a look towards adaptation and universal approximators. In To be presented at the 57th IEEE Conference on Decison and Control, Miami Beach, Florida, USA, 2018.

[19] M.Bin and L. Marconi. Adaptive post-processing internal models design for MIMO minimum-phase nonlinear systems. Under review.

[20] D. Astolfi, A. Isidori, and L. Marconi. Output regulation via low-power construction. In N. Petit, editor, Feedback Stabilization of Controlled Dynamical Systems, pages 143-165. Springer, 2017.

[21] M. W. Hirsch. Differential Topology. Springer-Verlag, 1994.

[22] J. Huang and W. J. Rugh. An approximation method for the nonlinear servomechanism problem. IEEE Trans. Autom. Control, 37(9):13951398, 1992.

[23] D. Astolfi, L. Praly, and L. Marconi. Approximate regulation for nonlinear systems in presence of periodic disturbances. In Proc. 54th IEEE Conference on Decision and Control, pages 7665-7670, 2015.

[24] F. Forte, L. Marconi, and A. R. Teel. Robust nonlinear regulation: Continuous-time internal models and hybrid identifiers. IEEE Trans. on Automat. Cont., 62(7):3136-3151, 2017.

[25] A. Isidori. Lectures in Feedback Design for Multivariable Systems. Springer International Publishing, 2017.

[26] D. Astolfi, A. Isidori, L. Marconi, and L. Praly. Nonlinear output regulation by post-processing internal model for multi-input multioutput systems. In Proc. 9th IFAC NOLCOS, pages 295-300, 2013.

[27] L. Wang, A. Isidori, H. Su, and L. Marconi. Nonlinear output regulation for invertible nonlinear MIMO systems. Int. J. of Robust and Nonlinear Control, 26:2401-2417, 2016.

[28] A. Serrani and A. Isidori. Global robust output regulation for a class of nonlinear systems. Systems \& Control Letters, 39:133-139, 2000. 